\title{
Johnson-Nyquist noise in films and narrow wires
}

\author{
Misha Turlakov \\ Department of Physics, University of Illinois, 1110 W. Green Street, Urbana, IL 61801
}

(October 26, 2018)

\begin{abstract}
The Johnson-Nyquist noise in narrow wires having a transverse size smaller than the screening length is shown to be white up to the frequency $D / L^{2}$ and to decay at higher frequencies as $\omega^{-\frac{1}{2}}$. In two-dimensional films having a thickness smaller than the screening length, the Johnson-Nyquist noise is predicted to be frequency independent up to the frequency $\sigma_{2 D} / L$ and to have a universal $1 / \omega$ spectrum at higher frequencies. These results are contrasted with the conventional noise spectra in neutral and three-dimensional charged liquids.
\end{abstract}

PACS numbers: 71.10.Ca, 72.70.+m, 72.30.+q, 05.40.Ca

It is interesting to analyze the differences between charged and neutral systems due to the long-range nature of the Coulomb interaction. The role of the Coulomb interaction depends crucially on the effective dimensionality of the charged system. For instance, in threedimensional systems Coulomb interaction transforms the gapless density excitations of neutral liquids (acoustic phonons) to gapped plasmons. Nevertheless, in one- and two-dimensional systems plasmons remain gapless. Here I examine the noise spectrum as another aspect of the singular role of Coulomb interaction on collective phenomena (plasmons, noise) which depends critically on the dimensionality.

The noise spectrum is quite different in charged and neutral liquids. The equilibrium Johnson-Nyquist noise $(\mathrm{JNN}) \mathrm{B}$ in an electrical conductor (with a screening length smaller than any size of the conductor) is independent of frequency (white noise) up to the very high frequency (the smaller of the elastic scatteripg rate $1 / \tau$ and the Maxwell relaxation frequency $4 \pi \sigma)^{2}$; while in neutral liquids, the noise becomes frequency-dependent above the "Thouless" frequency $D / L^{2}$ ( $D$ is a diffusion constant and $L$ is the distance between points). The difference is due to the screening in charged liquids and depends on the dimensionality of the conductor. I show here that for electrical wires having a transverse size (a) smaller than the screening length $\lambda_{D}$ (here referred to as "narrow" wires), the JNN decays as $\omega^{-\frac{1}{2}}$ above the "Thouless" frequency. Similarly, for two-dimensional films having a thickness smaller than the screening length the JNN decreases as $1 / \omega$ above the characteristic frequency $\sigma_{2 D} / L$.

To calculate the fluctuations of the electrochemical potential, we need to relate it to the coupled fluctuations of charge density and currents. We start by writing the continuity equation and the equation for the current valid in the hydrodynamic limit 3 :

$$
\frac{\partial \rho}{\partial t}+\operatorname{div}(\vec{j})=0 ; \quad \vec{j}=\sigma \vec{E}^{t o t}-D \vec{\nabla} \rho .
$$

For self-consistency, we need to account for the potential induced by the fluctuation of charge density: $\phi_{q, \omega}^{\text {ind }}=$ $u_{1}(q) \rho_{q, \omega}$ (Coulomb's law). If we consider a conductor with transverse dimensions $(a)$ smaller than the screening length $\lambda_{D}$, then $u_{1}(q)=2 \ln \frac{1}{q a}$ is a one-dimensional Coulomb potential ( $q$ is a wave vector along the onedimensional conductor). The total potential driving current is the sum of the external and induced potentials. Thus the full system of equations is:

$$
\begin{array}{r}
i \omega \rho_{q, \omega}+i q j_{q, \omega}=0, \quad j_{q, \omega}=(i q) \sigma \phi_{q, \omega}^{t o t}+(i q) D \rho_{q, \omega} \\
\phi_{q, \omega}^{t o t}=\phi_{q, \omega}^{e x t}+\phi_{q, \omega}^{i n d}, \quad \phi_{q, \omega}^{i n d}=2 \ln \frac{1}{q a} \rho_{q, \omega} .
\end{array}
$$

Finally, after some elementary algebra, we can use the above equations to relate the charge density variation to the external potential:

$$
\rho_{q, \omega}=-\frac{\sigma_{1} q^{2}}{-i \omega+\left(D+2 \sigma_{1} \ln (1 / q a)\right) q^{2}} \phi_{q, \omega}^{e x t},
$$

where $\sigma_{1}=\sigma a^{2}$ is a one-dimensional conductivity. The conductivity $\sigma$ and the diffusion constant $D$ are related by the Einstein formula $\sigma=D \chi_{0}$, where the static charge compressibility $\chi_{0}$ can be expressed through the Debye screening (or Thomas-Fermi) length $\chi_{0}=1 / 4 \pi \lambda_{D}^{2}$. The density-density response function $\chi_{q, \omega}$ is

$$
\chi_{q, \omega} \equiv \frac{\rho_{q, \omega}}{\phi_{q, \omega}^{e x t}}=-\frac{D \chi_{0} a^{2} q^{2}}{-i \omega+D q^{2}\left(1+2 a^{2} \chi_{0} \ln (1 / q a)\right)} .
$$

We can now apply the fluctuation dissipation theorem (FDT) to calculate the density fluctuation spectrum (assuming classical fluctuations, $\hbar \omega \ll k T)$ :

$$
<\left|\delta \rho_{q, \omega}\right|^{2}>=\frac{\hbar}{\pi} \operatorname{Im} \chi_{q, \omega} \operatorname{coth}\left(\frac{\hbar \omega}{2 k T}\right) \cong \frac{2 k T}{\pi \omega} \operatorname{Im} \chi_{q, \omega} .
$$

The induced potential fluctuations can be expressed through the charge density fluctuations:

$$
\begin{array}{r}
<\left|\phi_{q, \omega}^{\text {ind }}\right|^{2}>=\left(u_{1}(q)\right)^{2}<\left|\delta \rho_{q, \omega}\right|^{2}> \\
<\left|\phi_{q, \omega}^{\text {ind }}\right|^{2}>=\frac{2 k T}{\pi} \frac{\sigma_{1} q^{2}\left(2 \ln \frac{1}{q a}\right)^{2}}{\omega^{2}+D^{2}\left(1+\frac{a^{2}}{2 \pi \lambda_{D}^{2}} \ln \frac{1}{q a}\right)^{2} q^{4}} .
\end{array}
$$


We can compare this expression for the spectral density of noise in a 1D wire (Eqn.1) with the spectral density of potential fluctuations in bulk three-dimensional charged and neutral liquids. In the case of a three-dimensional charged liquid, we need to use the three-dimensional Coulomb potential $\phi_{q, \omega}^{\text {ind }}=u_{3}(q) \rho_{q, \omega}=\frac{4 \pi}{q^{2}} \rho_{q, \omega}$. Following the above simple derivation, we get the expression for voltage fluctuations (it is sufficient for our purposes to consider only longitudinal fluctuations) in a threedimensional conductort:

$$
<\left|\phi_{q, \omega}^{i n d(3 d)}\right|^{2}>=\frac{2 k T}{\pi q^{2}} \frac{16 \pi^{2} \sigma}{\omega^{2}+\left(D q^{2}+4 \pi \sigma\right)^{2}} .
$$

In the case of a neutral liquid, there is no long-range induced potential; therefore, we get the standard densitydensity response function and potential fluctuations describing diffusion:

$$
<\left|\phi_{q, \omega}^{(n)}\right|^{2}>=\frac{<\left|\delta \rho_{q, \omega}\right|^{2}>}{\chi_{0}^{2}}=\frac{2 T}{\pi \chi_{0}^{2}} \frac{D\left|\chi_{0}\right| q^{2}}{\omega^{2}+\left(D q^{2}\right)^{2}} .
$$

We can now use the spectral densities (Eqns. 1-30 to calculate the experimentally measured differential noise between the two ends of the sample, averaged over transverse modes:

$$
\begin{aligned}
<\left|\phi_{12}(\omega)\right|^{2}> & =\sum_{q_{x}} 4 \frac{\sin ^{2}\left(q_{x} a / 2\right)}{q_{x}^{2} a^{2}} \sum_{q_{y}} 4 \frac{\sin ^{2}\left(q_{y} a / 2\right)}{q_{y}^{2} a^{2}} \\
& \int \frac{d q_{z}}{2 \pi} 4 \sin ^{2}\left(\frac{q_{z} L}{2}\right)<|\phi(q, \omega)|^{2}>.
\end{aligned}
$$

The Johnson-Nyquist noise in a three-dimensional conductor (2) can be easily calculated, because the dominant contribution to the sums comes from the transverse zero mode $\left(q_{x}=q_{y}=0\right.$, corresponding to the uniform density of the liquid along the transverse directions):

$$
<\left|\phi_{12}^{3 d}(\omega)\right|^{2}>=\frac{2 k T}{\pi} \frac{L}{\sigma S} \frac{1}{1+\left(\frac{\omega}{4 \pi \sigma}\right)^{2}} .
$$

Such noise is readily interpreted as the noise from a conductor having an internal resistance $R=\frac{L}{\sigma S}$ and an internal capacitance $C=\frac{S}{4 \pi L}$ connected in parallello: $R(\omega)=R /\left(1+(R C \omega)^{2}\right)$. Remarkably, the JohnsonNyquist noise is white up to the frequency $4 \pi \sigma$, which is independent of the length of the wire. It is important to point out that the noise can depend on frequency through the frequency dependence of the conductivity $\sigma(\omega)$. For the Drude model of conductivity, the characteristic frequency for fall-off of the conductivity $\sigma(\omega)$ is then the elastic scattering rate $1 / \tau$.

In the case of a "one-dimensional" wire $a<\lambda_{D}$, we can take into account only one "zero mode" $\left(q_{x}=q_{y}=0\right)$, since higher harmonics contribute at frequencies of order $D / a^{2}>\sigma$. If we approximate the weak logarithmic dependence in Eqn.(11) by a constant $\ln \frac{1}{q a} \rightarrow \ln \frac{L}{a}$, we get an expression similar to Eqn.(3) with the renormalized diffusion coefficient $D^{\prime} \equiv D\left(1+\frac{a^{2}}{2 \pi \lambda_{D}^{2}} \ln (L / a)\right)$. Thus the frequency dependence of noise for a "one-dimensional" wire is the same as for a neutral liquid. This result is to be expected, since the screening is not efficient in one dimension. The integral over wave vector $q_{z}$ can be evaluated explicitly assuming for simplicity $a^{2} \ln (L / a) \gg 2 \pi \lambda_{D}^{2}$. The Johnson-Nyquist noise for a narrow wire is

$$
<\left|\phi_{12}^{1 d}(\omega)\right|^{2}>=2 k T \frac{L}{\sigma_{1} \theta}\left(1-e^{-\theta}(\cos \theta-\sin \theta)\right),
$$

where $\theta=\left(\omega / 2 \omega_{0}\right)^{1 / 2}$ and $\omega_{0}=D^{\prime} / L^{2}$ is the natural diffusion frequency. The expression in the bracket of Eqn. 6 is always positive as it must be, and the oscillating nature of the second term is due to the "resonant" contributions of the longitudinal "diffusion modes". From the above expression for noise in a one-dimensional wire, we see that it is approximately white up to the "Thouless" frequency $\omega_{0}$ and equal to $4 k T \frac{L}{\sigma_{1}}$. It decays above this frequency as $1 / \sqrt{\omega}$. The same frequency dependence (with $D^{\prime} \equiv D$ ) is expected for the fluctuations of the chemical potential between two points in a narrow vessel $\left(\omega \ll D / a^{2}\right)$ of neutral liquid. In fact, it is the classical result for any quantity (such as temperature, density) obeying a diffusion process that does not have long-range correlations.

The noise in a two-dimensional film $\left(a<\lambda_{D}, L_{z}, L_{\perp} \gg\right.$ $\left.\lambda_{D}\right)$ can be calculated likewise using the above formalism. The noise is measured along the $z$ direction, $L_{\perp}$ and $x$ are the transverse width and the transverse coordinate of the film, respectively, and $a$ is the thickness. Using the corresponding expressions for 2D Coulomb potential $u_{2}(q)=\frac{2 \pi}{q}$ and the two-dimensional conductivity $\sigma_{2 D}=\sigma a$, the spectral density of $2 \mathrm{D}$ noise is

$$
<\left|\phi_{q, \omega}^{i n d(2 d)}\right|^{2}>=\frac{2 k T}{\pi} \frac{4 \pi^{2} \sigma_{2 D}}{\omega^{2}+\left(D q^{2}+2 \pi \sigma_{2 D} q\right)^{2}} .
$$

Since the in-plane dimensions of the film are much larger than the screening length, we can simplify the denominator of the above equation by neglecting the term $D q^{2}$ (since $q \ll \frac{\sigma_{2 D}}{D}=\frac{a}{4 \pi \lambda_{D}} \frac{1}{\lambda}$, then $D q^{2} \ll \sigma_{2 D} q$ ). The differential noise between the two ends of the twodimensional strip, averaged over the transverse modes, is

$$
\begin{array}{r}
<\left|\phi_{12}^{(2 d)}(\omega)\right|^{2}>=\int \frac{d q_{z}}{2 \pi} 4 \sin ^{2}\left(\frac{q_{z} L_{z}}{2}\right) \\
\sum_{q_{x}} 4 \frac{\sin ^{2}\left(q_{x} L_{\perp} / 2\right)}{q_{x}^{2} L_{\perp}^{2}}<\left|\phi_{q, \omega}^{i n d(2 d)}\right|^{2}>.
\end{array}
$$

The integration (if the sum can be approximated by the integral) over the transverse dimension $x$ can be done exactly, and we get the expression:

$$
<\left|\phi_{12}^{(2 d)}(\omega)\right|^{2}>=\frac{2 k T}{\pi \sigma_{2 D}} \int \frac{d q_{z}}{2 \pi} 4 \sin ^{2}\left(\frac{q_{z} L_{z}}{2}\right)
$$




$$
\begin{aligned}
& \int \frac{d q_{x}}{2 \pi} 4 \frac{\sin ^{2}\left(q_{x} L_{\perp} / 2\right)}{q_{x}^{2} L_{\perp}^{2}} \frac{1}{\left(b^{2}+q_{z}^{2}\right)+q_{x}^{2}}= \\
& =\frac{2 k T}{\pi \sigma_{2 D}} \int \frac{d q_{z}}{2 \pi} 4 \sin ^{2}\left(\frac{q_{z} L_{z}}{2}\right) F\left(q_{z}\right) .
\end{aligned}
$$

$F\left(q_{z}\right) \equiv \frac{1}{2 L_{\perp}^{2}\left(q_{z}^{2}+b^{2}\right)}\left(2 L_{\perp}-\frac{1-\exp \left(-2 L_{\perp} \sqrt{q_{z}^{2}+b^{2}}\right)}{\sqrt{q_{z}^{2}+b^{2}}}\right)$

where $b \equiv \frac{\omega}{2 \pi \sigma_{2 D}}$ is the inverse of the characteristic length scale of the problem. The limiting expressions for the function $F\left(q_{z}\right)$ are:

$$
F\left(q_{z}\right) \simeq \begin{cases}\frac{1}{L_{\perp}\left(q_{z}^{2}+b^{2}\right)} & \text { if } \sqrt{q_{z}^{2}+b^{2}} L_{\perp} \gg 1 \\ \frac{1}{\sqrt{q_{z}^{2}+b^{2}}} & \text { if } \sqrt{q_{z}^{2}+b^{2}} L_{\perp} \ll 1\end{cases}
$$

The integral over wave vector $q_{z}$ can be taken in such limiting cases. But a careful analysis shows that for the case $\sqrt{q_{z}^{2}+b^{2}} L_{\perp} \ll 1$ the summation over the transverse modes $q_{x}$ cannot be approximated by the integral. The main contribution actually comes from the "zero mode" $q_{x}=0$ in spite of the condition $L_{\perp} \gg \lambda_{D}$. Taking the above considerations into account, the answer for the noise across two-dimensional film is given below.

$$
<\left|\phi_{12}^{(2 d)}(\omega)\right|^{2}>\simeq \frac{2 k T}{\pi \sigma_{2 D}} * \begin{cases}\frac{\sigma_{2 D}}{\omega L_{\perp}} & \text { if } \omega \gg \frac{2 \pi \sigma_{2 D}}{L_{z}}, \frac{\sigma_{2 D}}{L_{\perp}} \\ \frac{L_{z}}{L_{\perp}} & \text { if } \omega \ll \frac{\sigma_{2}\left(D_{z}\right.}{\max \left(L_{z}, L_{\perp}\right)}\end{cases}
$$

In some cases, the integral can be expressed through Bessel functions, but only the final asymptotic expressions are of interest here. As pointed above when $L_{\perp} \ll$ $\frac{\sigma_{2 D}}{\omega}$, the main contribution (after the integration over $q_{z}$ ) calculated from the "zero mode" $q_{x}=0$ is proportional to $L_{z} / L_{\perp}$, while the estimate of the integral over the higher harmonics of $q_{x}$ is smaller and proportional to $\ln \frac{L_{z}}{\lambda_{D}}$ (if $L_{z} \gg L_{\perp}$ ). At the frequencies of the order of the 3D Maxwell frequency, the film cannot be considered as two-dimensional and transverse harmonics other than the "zero-frequency" one $\left(q_{y}=0\right)$ must be taken into account.

It is very interesting that for frequencies above $\frac{\sigma_{2 D}}{L_{\perp, z}}=$ $\sigma \frac{a}{L_{\perp, z}}$ frequency and below the Maxwell relaxation frequency $4 \pi \sigma$ the noise is universal (independent of the material specific conductivity $\sigma_{2 D}$ and dependent only on the transverse size $L_{\perp}$ ) and equal to $\frac{4 k T}{\omega L_{\perp}}$.

By the FDT the noise is proportional to the total dissipation which is the product of the dissipation per unit length and the characteristic dissipative length scale. The dissipation per unit length is inversely proportional to the material specific conductivity $\sigma_{2 D}$. At the low frequencies, the dissipative length scale is set by the longitudinal size $L_{z}$ of the sample, therefore the noise is proportional to $L_{z} / \sigma_{2 D}$. At the high frequencies, as soon as the length scale $\sigma_{2 D} / \omega$ becomes smaller than the longitudinal size $L_{z}$, the dissipative length scale is set by this length $\sigma_{2 D} / \omega$. Therefore the high frequency noise becomes independent of the conductivity. The universality of the noise at high frequencies is special to the two-dimensional situation and is due to dimensional reasons. Only in two dimensions the length scale is given by the simple ratio $\sigma_{2 D} / \omega$ of the conductivity $\sigma_{2 D}$ (or the conductance) and the frequency $\omega$.

At the low frequencies $(\omega \rightarrow 0)$, the noise has the standarfform consistent with the fluctuation-dissipation theorem $1 \mathrm{~d}$ applied to the whole sample

$$
<\left|\phi_{12}^{(2 d)}\right|^{2}>\simeq \frac{2}{\pi} k T R,
$$

where $R=\frac{L_{z}}{\sigma_{2 D} L_{\perp}}$ is the dc resistance of the film. The noise in the 3D wire (Eqn. 5) and the 1D wire (Eqn. 6) is consistent with the FDT as well.

The fluctuation-dissipation theorem applied to the whole sample relates the voltage noise between the ends of the sample to the real part of the impedance $Z(\omega)$ of the sample. At zero frequency the capacitive part of the impedance is always short-circuited by the dissipative part (the resistance). The resistance of the wire in all considered cases is expressed through the geometrical sizes, as it can be seen from the Eqns. (5,6,99). If the resistance is measured from the zero frequency expression of the noise, then the effective capacitance $C$ of the sample can be measured from the high-frequency $(\omega R C \gg 1)$ expression of the noise: $\operatorname{Re} Z(\omega) \simeq \frac{R}{(\omega R C)^{2}}$. We can use this equation to interpret the high-frequency noise in the $1 \mathrm{D}$ wire and the 2D strip and to write the expressions for the effective capacitances of the corresponding wires. In case of the 3D wire (with the well screened Coulomb interaction), the sample has a constant (frequency independent) capacitance $C=\frac{S}{4 \pi L}$. The effective capacitance of the $2 \mathrm{D}$ strip at the high frequencies $\left(\sigma \gg \omega \gg \sigma_{2 D} / L_{z}\right)$ is

$$
C_{2 D} \simeq L_{\perp} \sqrt{\frac{\sigma_{2 D}}{\omega L_{z}}}=\frac{L_{\perp} a}{L_{z}} \sqrt{\frac{\sigma L_{z}}{\omega a}} \gg \frac{L_{\perp} a}{L_{z}} .
$$

The effective capacitance of the $1 \mathrm{D}$ wire at the frequencies $\left(\sigma \gg \omega \gg D / L^{2}\right)$ is

$$
\begin{aligned}
& C_{1 D} \simeq\left(\frac{\sigma}{\omega}\right)^{3 / 4} \frac{a^{2}}{2 \pi^{3 / 4} \lambda_{D}^{1 / 2} L^{1 / 2}}= \\
& =\frac{1}{2 \pi^{3 / 4}}\left(\frac{L}{\lambda_{D}}\right)^{1 / 2}\left(\frac{\sigma}{\omega}\right)^{3 / 4} \frac{a^{2}}{L} \gg \frac{a^{2}}{L} .
\end{aligned}
$$

In the both $1 \mathrm{D}$ and $2 \mathrm{D}$ wires, the effective capacitances are frequency dependent and much larger than the standard geometrical capacitances, because the Coulomb interaction is not completely screened and non-local.

Since the noise has a frequency dependent form, by FDT it implies the same frequency dependence of the real part of the impedance $Z(\omega)$. The measurement of the complex impedance can be more straightforward way to access the predicted frequency dependencies of noise than a direct measurement of noise. 
The nature of the relaxation of a random potential fluctuation is quite different in charged and neutral liquids. In charged three-dimensional liquids, it is essentially the fast process of screening, and in neutral liquids it is the process of diffusion. The appropriate physical picture of fluctuations in a three-dimensional electrical conductor is that charge fluctuations relax on a very fast time scale $1 / 4 \pi \sigma$, producing quasi-homogeneous current fluctuations. In one-dimensional systems such as narrow wires, the Coulomb interaction does not cause long-range correlations; therefore, the noise in a narrow conductor is similar to the noise spectra in neutral systems. The difference in the chemical potential between two points is relaxed through diffusion on a characteristic time scale $L^{2} / D$ quadratically dependent on the length $L$ between points. The situation of two-dimensional noise is intermediate, and the characteristic time scale $L / \sigma_{2 D}$ of the relaxation of the voltage fluctuation difference between two points is linearly dependent on the distance $L$.

The noise spectrum and the spectrum of collective modes are closely related. Since the spectrum of collective modes (plasmons) is given by the zeros of the dielectric constant $\epsilon(q, \omega)$, they give rise to the dominant contribution to the noise spectrum which is proportional to the $\operatorname{Im} \frac{1}{\epsilon(q, \omega)}$ (see the comment 5 ). At the end, both the frequency dependence of the noise and the dispersion of the collective modes depend essentially only on the effective dimensionality of the Coulomb interaction.

The experimental observation of the predicted noise properties is feasible in semiconducting materials having a low carrier concentration.11 The screening length $\lambda_{D}$ in such materials 12 can be as large as $10^{-4} \mathrm{~cm}$. In metals, both the elastic rate $1 / \tau$ and the Maxwell frequency $4 \pi \sigma$ are high and difficult to observe, while the typical screening length for a metal is of order of $10^{-8} \mathrm{~cm}$. In fact, with current experimental techniques (see Reference 13 for a review of experiments), even the high Maxwell relaxation frequency crossover $4 \pi \sigma$ can be observed in "wide" wires ( $a \gg \lambda_{D}$, the situation almost always encountered) with poor conductivity. By a convenient choice of the mobilities of the semiconductor materials and their size $L$, the "Thouless frequency" $D / L^{2}$ and the two-dimensional "relaxation frequency" $\sigma_{2 D} / L$ should be accessible. Several other experimental low-dimensional systems satisfying the condition of the absence of screening can be suggested.

The contacts to the external leads (and associated boundary conditions) are not considered explicitly in this paper. It is assumed that the main source of noise is the bulk of a wire or a film, and the contacts have a resistance much lower than a bulk system.

The question of the frequency dependence of equilibrium and "shot" noise was raised recently by Y. Naveh et al 13 Special geometries with external screening were suggested to observe the Maxwell and Thouless crossover frequencies. The above calculation shows that the crossover at the Maxwell relaxation frequency is a general prop- erty of Coulomb systems and should be observed independently of geometry and length $L$ for "wide" wires. Moreover, for "narrow" wires $\left(a<\lambda_{D}\right)$ the Thouless frequency crossover should be seen independently of "external screening" by electrodes or the ground plane.

In conclusion, the noise in narrow wires $\left(a<\lambda_{D}\right)$ becomes frequency-dependent starting from the low frequency $D / L^{2}$ (quite similar to simple diffusion systems), although in wide conductors, the noise is white up to the smaller of the frequencies $4 \pi \sigma$ or $1 / \tau$. In two-dimensional films, the Johnson-Nyquist noise has a universal $1 / \omega$ spectrum in the wide range of frequencies $\sigma \gg \omega \gg \sigma \frac{a}{L}$.

This work was supported by the National Science Foundation through the Science and Technology Center for Superconductivity (Grant No. DMR-91-20000). I thank A. Leggett and M. Weissman for helpful discussions. I am grateful to A. Leggett, R. Ramazashvili and H. Westfahl for the useful remarks and the careful reading of the manuscript.

${ }^{1}$ M.B. Johnson, Phys. Rev. 29, 367 (1927), H. Nyquist, Phys. Rev. 32, 110 (1928).

2 The Maxwell frequency $4 \pi \sigma$ can be expressed through the three-dimensional plasma frequency $\omega_{p}$ and the elastic scattering time $\tau: 4 \pi \sigma=\omega_{p}^{2} \tau$. The threshold frequencies $D / L^{2}$ and $\sigma_{2 D} / L$ are much smaller than the three-dimensional Maxwell frequency $4 \pi \sigma: 4 \pi \sigma \gg D / L^{2}=4 \pi \sigma\left(\lambda_{D}^{2} / L^{2}\right)$ (1D), $4 \pi \sigma \gg \sigma_{2 D} / L=4 \pi \sigma(a /(4 \pi L))(2 \mathrm{D})$. These threshold frequency $D / L^{2}$ and $\sigma_{2 D} / L$ can be made arbitrarily small by increasing the separation between two points $L$.

${ }^{3}$ D. Pines, Ph. Noziéres, The theory of quantum liquids (Benjamin, New York, 1966). For brevity, we omit the sign $\delta$ of small variations in all expressions.

${ }^{4}$ Except ultra-low temperatures, the Johnson-Nyquist noise is classical at the frequencies of interest (e.g. a temperature $k T=0.1 K$ corresponds to a frequency $1.6 \mathrm{GHz}$ ).

${ }^{5}$ Equation (2) can be also derived in two other equivalent ways, elucidating the meaning of charge and current fluctuations in a charged liquid. One way is to introduce stochastic current source $j^{a c}$ into the current equation 8 : $j_{q, \omega}=\sigma(i q) \phi_{q, \omega}+D(i q) \rho_{q, \omega}+j^{a c}$. This derivation illustrates the role of quasi-homogeneous current fluctuations effectively screening charge fluctuations. Yet a third derivation explicitly demonstrates the role of screening. Since $\delta \phi_{q, \omega}^{t o t}=\frac{4 \pi}{\epsilon(q, \omega) \epsilon_{0} q^{2}} \delta \rho_{q, \omega}$, the fluctuations of $\delta \phi_{q, \omega}^{t o t}$ due to the FDT are given by the expression $\left\langle\left|\delta \phi_{q, \omega}^{\text {tot }}\right|^{2}\right\rangle=$ $\frac{\hbar}{\pi} \operatorname{Im} \frac{u_{3}(q)}{\epsilon(q, \omega)} \operatorname{coth} \frac{\omega}{2 k T}$. The dielectric constant $\epsilon(q, \omega)$ can be represented in the hydrodynamic limit 3 as: $\epsilon(q, \omega)=1-$ $4 \pi \chi^{(n)}(q, \omega) / q^{2}=1+4 \pi \sigma /\left(i \omega+D q^{2}\right)$.

${ }^{6}$ S.M. Rytov, Yu.A. Kravtsov, and V.I. Tatarskii, Principles of Statistical Radiophysics, v.3 (Springer-Verlag, Berlin, 1976), p. 167.

${ }^{7}$ All calculations here are done in the assumption of a quasi- 
stationary condition ( the length of a conductor $L$ is much smaller than the electromagnetic wavelength $\left.\lambda=c \frac{2 \pi}{\omega}\right)$. This condition implies the absence of the retardation effects for an electromagnetic field profile inside a conductor (it does not imply the homogeneity of electric field inside a conductor due to screening and diffusion processes).

${ }^{8}$ Sh. Kogan, Electronic noise and fluctuations in solids, (Cambridge University Press, Cambridge, 1996); B.L. Altshuler, A.G. Aronov, and D.E. Khmelnitsky, J. Phys. C 15, 7367-86 (1982).

${ }^{9}$ R. Voss, J. Clark, Phys. Rev. B 13, 556-573 (1976).

${ }^{10}$ H.B. Callen, T.A. Welton, Phys. Rev. B 83, 34-40 (1951).

${ }^{11}$ It is important to point out that the condition $a<\lambda_{D}$ can be satisfied, while "weak localization" and Luttinger-liquid physics are not relevant for the problem discussed here. If the electron Fermi-wavelength $\lambda_{F}$ is much smaller than the diameter $a$ (many transverse channels), the Luttinger liquid physics is not relevant. If either $\sigma_{1}=\frac{D}{4 \pi \lambda_{D}^{2}} \frac{\pi a^{2}}{L} \gg e^{2} / \hbar$ (or equivalently $L \ll L_{l o c}=\frac{\hbar}{e^{2}} D \frac{a^{2}}{2 \lambda_{D}^{2}}$ ) or $L_{\phi}, L_{T} \ll a$ (the phase coherence length $L_{\phi}$, the thermal length $L_{T}$ ), than the "weak localization" corrections are small.

12 The large high-frequency dielectric constant $\epsilon_{\infty}$ of a material is favorable for the experimental observation, since the screening length $\tilde{\lambda_{D}}$ becomes larger $\left(\tilde{\lambda_{D}}{ }^{2}=\epsilon_{\infty} \lambda_{D}^{2}\right)$. The threshold frequencies become smaller (e.g. $\tilde{\omega}_{p}^{2}=\omega_{p}^{2} / \epsilon_{\infty}$ ).

${ }^{13}$ Y. Naveh, D. Averin, and K. Likharev, Phys. Rev. Lett. 79, 3482 (1997), Phys. Rev. B 59, 2848-60 (1999). 